\title{
ÁGUAS MODAIS SUBTROPICAIS DO ATLÂNTICO SUL NA BASE LEVITUS
}

\author{
FELIX, C. ${ }^{1}$; GABIOUX, M. ${ }^{1}$ \& PAIVA, A.M. ${ }^{2}$ \\ 1PEnO/COPPE, ${ }^{2}$ PEnO/COPPE, DENO/EP \\ Universidade Federal do Rio de Janeiro- Rio de Janeiro \\ Cx. Postal 68508, RJ, 21945-970, Brasil
}

\begin{abstract}
Felix, C.; Gabioux, M. \& Paiva, A.M. 2005. South atlantic subtropical mode waters in the Levitus database. Braz. J. Aquat. Sci. Technol. 9(2):17-22. ISSN 1808-7035. Mode waters are layers of vertically homogeneous water found over a large geographical area, occur within or near the top of the permanent pycnocline. The mode waters are characterized by the conservation of their properties of the formation area (temperature, salinity, potential vorticity). In South Atlantic Ocean, the subtropical mode waters are formed within the Brazil-Malvinas Confluence region, and keep their properties along the subtropical gyre. The objective of this work is to identify and to characterize subtropical mode waters from data of temperature and salinity of the Levitus database. The applied methodology consisted on the construction and analysis of zonal and meridional sections of potential temperature and salinity and volumetric T-S diagrams. Two mode waters were identified on the analysis, one with potential temperature (q) $\sim 14-16^{\circ} \mathrm{C}$ and another one with $q \sim 11-12^{\circ} \mathrm{C}$. These results were verified with information of hydrographic data collected presented in literature and will be used for future comparison with numerical models simulation.
\end{abstract}

Keywords: Subtropical mode waters, Levitus database, SACW.

\section{INTRODUÇÃO}

Águas Modais são camadas de água cuja principal característica é a homogeneidade vertical adquirida durante sua formação, através da convecção do inverno (Provost et al.,1999). Localizadas na região da picnoclina permanente e com extensa distribuição geográfica, caracterizam-se por conservar as propriedades da sua região de formação (temperatura potencial, salinidade, vorticidade potencial, ...). Águas modais podem ser identificadas como termostatas ou picnostatas em seções verticais de temperatura e salinidade, respectivamente, como mínimos de vorticidade potencial na coluna d'água, ou ainda como máximos de volume em diagramas T-S volumétricos.

A ocorrência de águas modais tem sido verificada em diversas bacias oceânicas. No Atlântico Norte, os três maiores volumes em T-S volumétrico são: Água Modal Subtropical do Atlântico Norte, também conhecida como Água de Dezoito Graus $\left(18^{\circ} \mathrm{C}\right)$; Água Modal Madeira e Água Modal Subpolar, cuja variedade mais densa é a Água do Mar de Labrador (McCartney \& Talley, 1982). No Pacífico Norte são encontradas a Água Modal Subtropical do Pacífico Norte, a Água Modal Central do Pacífico Norte, e descoberta mais recentemente, a Água Modal Subtropical do Leste do Pacífico Norte (Hosoda et al., 2001). Para o Pacífico Sul, foram identificadas duas águas modais: a Água Modal do Pacífico Sul e a Água Modal do Leste do Pacífico Sul (Hanawa \& Talley, 2001). No Oceano Índico, encontra-se a Água Modal
Subtropical do Oceano Índico e a Água Modal Subantártica do Sudoeste do Índico (Hanawa \& Talley, 2001).

No Atlântico Sul, a ventilação da termoclina permanente em diferentes regiões de formação produz a Água Modal Subtropical e a Água Modal Subantártica (Maamaatuaiahutapu et al.,1999). A primeira é associada com o contorno oeste do giro subtropical (Hanawa \& Talley, 2001) e pode ser separada da água modal subantártica seguindo a classificação de Provost et al. (1999), na qual a água modal subtropical é mais "leve" do que $27 \mathrm{~s}$, enquanto que a água subantártica se apresenta mais "pesada" (Maamaatuaiahutapu et al.,1999). Provost et al. (1999) identificaram, no Atlântico Sul, três tipos diferentes de águas modais subtropicais (Subtropical Mode Water - STMW) que constituem a Água Central do Atlântico Sul (ACAS). A primeira (STMW1) com densidade potencial $\left(s_{q}\right)$ de 26,2 se localiza a $\sim 100 \mathrm{~m}$ de profundidade, a segunda (STMW2), com $26,5 \mathrm{~s}$, entre 150 e $400 \mathrm{~m}$ e a terceira (STMW3), com s igual a 26,7 , entre 400 e $600 \mathrm{~m}$.

O objetivo do presente trabalho é identificar e caracterizar as águas modais subtropicais do Atlântico Sul, a partir dos dados de temperatura potencial e salinidade da base Levitus (1982). Esta base é composta por uma climatologia mensal de temperatura e sazonal de salinidade, com valores interpolados verticalmente para profundidades pré-estabelecidas e para $1^{\circ}$ de latitude-longitude. A escassez de dados hidrográficos no Atlântico Sul e a utilização generaliza- 
da desta base para inicialização de modelos numéricos de circulação em estudos climáticos serviram de motivação para o presente estudo. Apesar das limitações que o processo de interpolação espacial e temporal impõe à base Levitus, é importante verificar até que ponto ela preserva importantes feições do campo de massa na região da termoclina, especificamente suas águas modais.

\section{MATERIAL E MÉTODOS}

A área de estudo compreende duas sub-regiões do Oceano Atlântico Sul (Fig. 1): Região Oeste (latitudes 45,5 a $25,5^{\circ} \mathrm{S}$ e longitudes 55,5 a $10,5^{\circ} \mathrm{W}$ ), incluindo a confluência Brasil-Malvinas, onde as águas modais subtropicais são formadas; e a Região Leste (latitudes 40,5 a $25,5^{\circ} \mathrm{S}$ e longitudes $10,5^{\circ} \mathrm{W}$ a $10,5^{\circ} \mathrm{E}$ ), que inclui possíveis contribuições do oceano Índico, via Corrente das Agulhas (Hanawa \& Talley, 2001).

Foram utilizados os dados de temperatura, convertida para temperatura potencial (q) e salinidade (s) da base Levitus (1982) para o final de inverno austral, época em que a camada de mistura atinge as máximas profundidades. As águas modais foram identificadas a partir de termostatas e halostatas observadas em seções meridionais e zonais de q e $\mathrm{S}$, traçadas para todo o domínio de estudo, do cálculo de gradientes verticais mínimos de q, e de diagramas TS volumétricos.

Para cálculo dos TS volumétricos, foram consideradas apenas profundidades superiores a $100 \mathrm{~m}$, de forma a minimizar a influência de águas presentes na camada de mistura.

\section{RESULTADOS}

Na Região Oeste, observou-se uma água modal com q $~ 14-16^{\circ} \mathrm{C}$ e $\mathrm{S} \sim 35,4-35,9$, localizada entre latitudes 28,5 e $37,5^{\circ} \mathrm{S}$ e longitudes 47,5 e $12,5^{\circ} \mathrm{W}$, a uma profundidade média de $\sim 250 \mathrm{~m}$ e com espessura de $\sim 150 \mathrm{~m}$. Esta água foi identificada nas seções meridionais e zonais como uma termostata (Fig. 2a) e uma halostata (Fig. 2b) em toda sua extensão geográfica, e no TS volumétrico como um núcleo centrado em $q$ $\sim 15^{\circ} \mathrm{C}, \mathrm{S} \sim 35,5$ e $_{\mathrm{q}} \sim 26,3-26,4$ (Fig. 5a). Observou-se ainda nesta mesma região, uma variação zonal de qe $S$ dentro dos intervalos acima, com maiores valores na porção mais à oeste, decrescendo em direção a leste.

Ainda na Região Oeste, um volume de água com $q \sim 11-12^{\circ} \mathrm{C}$ e $S \sim 34,9$ - 35,4 foi localizado entre latitudes 24,5 e $31,5^{\circ} \mathrm{S}$ e longitudes 43,5 e $48,5^{\circ} \mathrm{W}$, com espessura máxima de $\sim 120 \mathrm{~m}$ e profundidade média de $\sim 450 \mathrm{~m}$. Este volume de água caracterizou-se pela presença de uma termostata (Fig.3a) e ausência de halostatas (Fig.3b), e por não apresentar uma conexão bem definida com a camada de mistura. No diagrama TS volumétrico (Fig.5a), apresentou-se como um núcleo centrado em $q \sim 11,0^{\circ} \mathrm{C}, \mathrm{S} \sim 35,0$ e $\mathrm{s}_{\mathrm{q}} \sim 26,7-26,8$.

Na Região Leste observou-se uma água modal com q 14-15 ${ }^{\circ} \mathrm{C}$ (Fig 4a), localizada entre latitudes 30,5 e $32,5^{\circ} \mathrm{S}$ e longitudes $3,5^{\circ} \mathrm{W}$ e $8,5^{\circ} \mathrm{E}$, a $\sim 250 \mathrm{~m}$ de

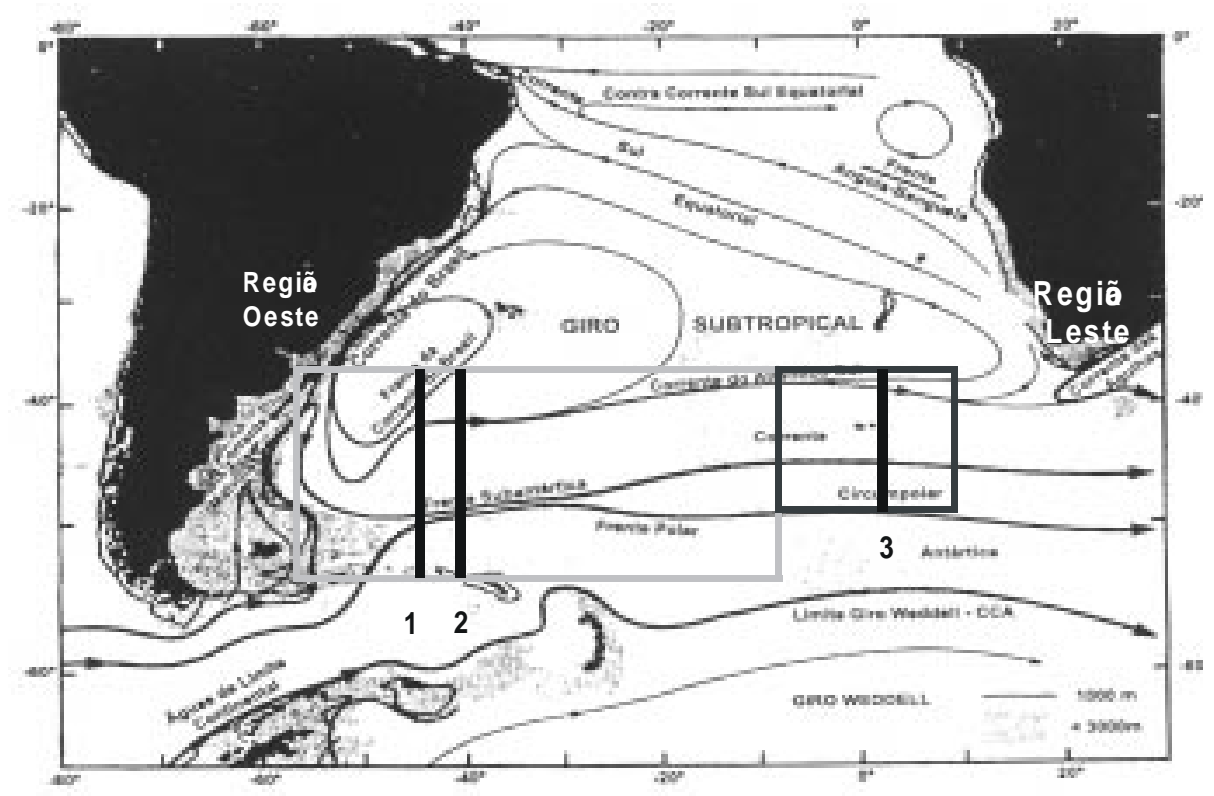

Figura 1 - Localização das áreas de estudo. O quadrado mais claro corresponde a Região Oeste, e o quadrado mais escuro, a Região Leste. As linhas pretas representam seções meridionais: (1) $45,5^{\circ} \mathrm{W}$, (2) $40,5^{\circ} \mathrm{W}$ e (3) $2,5^{\circ} \mathrm{E}$. De acordo com Peterson e Stramma (1991). Fonte: Silveira et al. (2000). 
profundidade e com espessura de $\sim 100 \mathrm{~m}$. Esta água apresentou duas halostatas: a primeira de $S \sim 35,1-35,3$, próxima ao meridiano zero e a segunda, mais salina, com $S \sim 35,2-35,5$ entre $\sim 2,5-8,5^{\circ} \mathrm{E}$ (Fig. 4b). No TS volumétrico (Fig.5b), esta água apareceu como um núcleo pouco evidente centrado em q $\sim 14,25^{\circ} \mathrm{C}, \mathrm{S} \sim 35,35$ e $s_{\mathrm{q}} \sim 26,41$.

Ainda na Região Leste, uma água de $q \sim 11-12^{\circ} \mathrm{C}$ e $S \sim 34,7$ - 35,0 localizou-se entre latitudes $\sim 28,5$ e $32,5^{\circ} \mathrm{S}$ e longitudes $\sim 2,5$ e $7,5^{\circ} \mathrm{E}$, a uma profundidade média de $\sim 500 \mathrm{~m}$, com espessura de $\sim 100 \mathrm{~m}$. Esta água caracterizou-se por apresentar uma termostata bem definida mas sem a presença de halostatas e, no TS volumétrico (Fig.5b), por um núcleo deslocado para $q \sim 10,75^{\circ} \mathrm{C}, \mathrm{S} \sim 34,95 \mathrm{e} \mathrm{s}_{\mathrm{q}} \sim 26,78$.

\section{DISCUSSÃO}

Das três águas modais subtropicais classificadas por Provost et al.(1999) no Atlântico Sul, apenas a STMW1 (q $16-18^{\circ} \mathrm{C}$ ) não foi observada na base Levitus. Sua existência pode mesmo ser questionada, uma vez que foi identificada por estes autores em dados hidrográficos de verão e de final de primavera, ou seja, em profundidades alcançáveis pela camada de mistura no inverno. Termostatas com esta faixa de temperatura foram somente identificadas, na base Levitus, para o verão e primavera.

A STMW2 apresentou-se nas duas sub-regiões como uma água modal de $q \sim 14-16^{\circ} \mathrm{C}$ claramente identificada na base Levitus, abrangendo uma grande área do Atlântico Sul em subsuperfície e conectada à camada de mistura. Essa conexão evidencia o processo de formação dessa água por subducção, que é a transferência de água da camada de mistura do final do inverno para a termoclina principal (Williams et al., 1995) e acontece em função do ciclo sazonal da camada de mistura, responsável também pelo processo de ventilação da termoclina principal (Marshall et al., 1993).

A STMW3 apresentou-se, na Região Leste, como mais fria $\left(q \sim 11-12^{\circ} \mathrm{C}\right)$ e menos salina $(\mathrm{S} \sim 34,7-$ $35,0)$ na base Levitus do que nos resultados de Provost et al.(1999). Na Região Oeste, foi encontrado um volume de água ( $q \sim 11-12^{\circ} \mathrm{C}$ ) parcialmente isolado da subsuperfície, sem conexão clara com a camada de mistura. Deste modo, o mecanismo clássico de formação por subducção, a partir da base da camada de mistura, não fica evidenciado e a classificação deste volume de água como água modal na Região Oeste é portanto questionável.

As águas modais subtropicais apresentam uma constância de temperatura em diferentes bacias oceânicas (Roemmich \& Cornuelle, 1992). A partir da base Levitus, STMW2 é a água modal subtropical dominante no Atlântico Sul, devido ao seu volume e distribuição maiores que STMW1 (observada apenas no verão e primavera) e STMW3. Uma água modal com temperatura semelhante a STMW2 pode ser encontrada no Pacífico Sul, neste caso com temperaturas ligeiramente mais elevadas $\left(\mathrm{q} \sim 15-17^{\circ} \mathrm{C}\right.$ ) entre as latitudes $30 \mathrm{e}$ $35^{\circ} \mathrm{S}$ (Roemmich \& Cornuelle, 1992), e no Pacífico
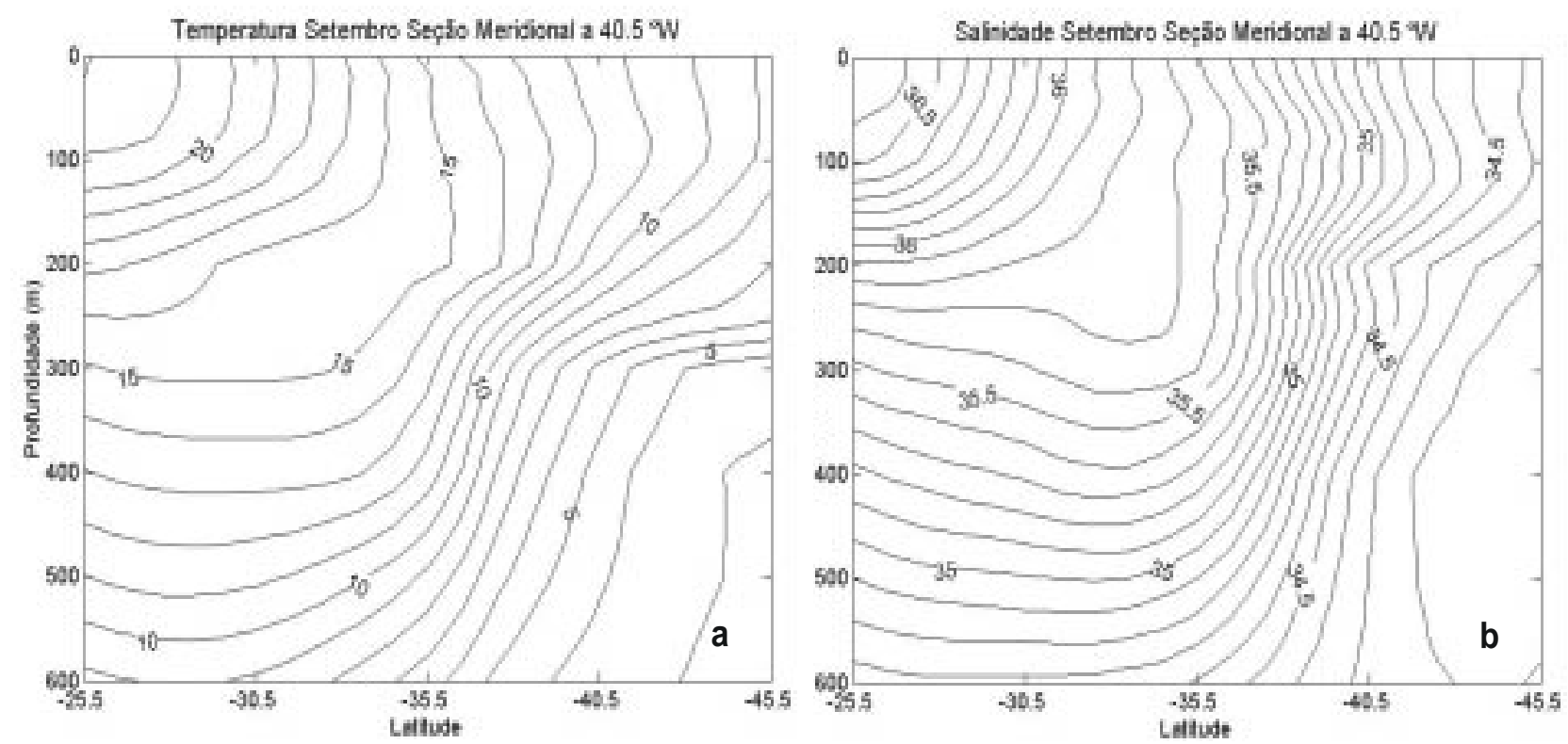

Figura 2 - Seção Meridional a 40,5 $\mathrm{W}$ (Região Oeste) entre $45,5^{\circ} \mathrm{S}$ e $25,5^{\circ} \mathrm{S}$ de (a) temperatura potencial $\left({ }^{\circ} \mathrm{C}\right)$ com intervalo de $1^{\circ} \mathrm{C}$ e $($ b) Salinidade com intervalo de 0,1 . 
Norte, no qual o núcleo da água modal subtropical se encontra a $q \sim 16.5^{\circ} \mathrm{C}$ (Hanawa \& Talley, 2001). No Atlântico Norte, a água modal subtropical dominante é a Água de Dezoito Graus ( $q \sim 18^{\circ} \mathrm{C}$ ), que se distribui na parte noroeste do giro subtropical (Hanawa \& Talley, 2001) em profundidades abaixo das alcançadas pela camada de mistura.

Em algumas regiões do Atlântico Sul, entre 25,5 e $40,5^{\circ} \mathrm{S}$ e 39,5 e $24,5^{\circ} \mathrm{W}$, observou-se inversões de densidade na coluna d'água que poderia originar-se de uma inconsistência entre as bases de dados de qe $S$ (maior número de dados de q provenientes de XBT). Este fato afeta a confiabilidade dos cálculos de densidade potencial e, conseqüentemente, optou-se pela não utilização de mínimos de vorticidade potencial na identificação das águas modais. Estão sendo investigadas ainda questões associadas a possível "bias" na base Levitus, em função de sua discretização vertical, o que
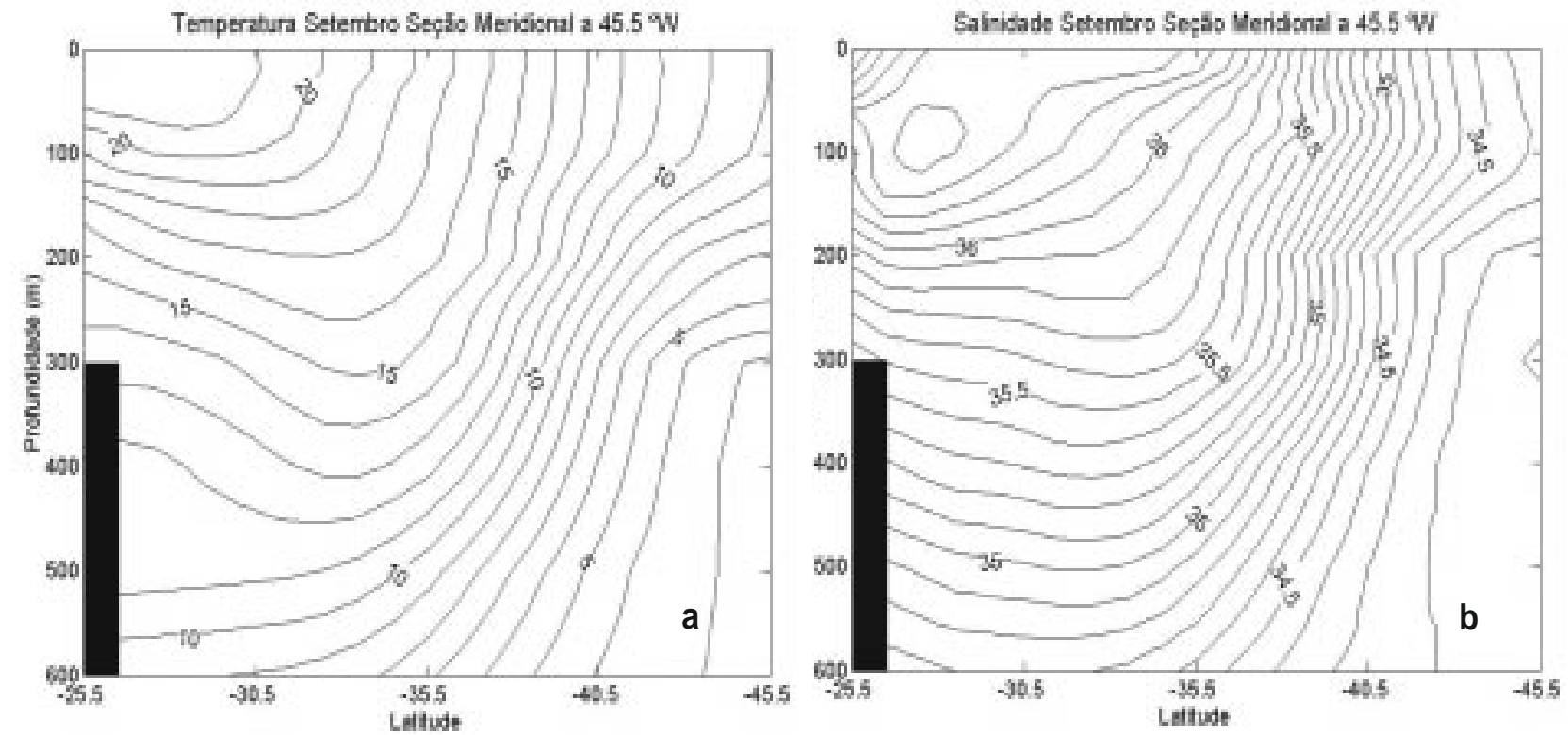

Figura 3 - Seção Meridional a $45,5^{\circ} \mathrm{W}$ (Região Oeste) entre $45,5^{\circ} \mathrm{S}$ e $25,5^{\circ} \mathrm{S}$ de (a) temperatura potencial $\left({ }^{\circ} \mathrm{C}\right)$ com intervalo de $1^{\circ} \mathrm{C}$ e $(\mathrm{b})$ Salinidade com intervalo de 0,1 .
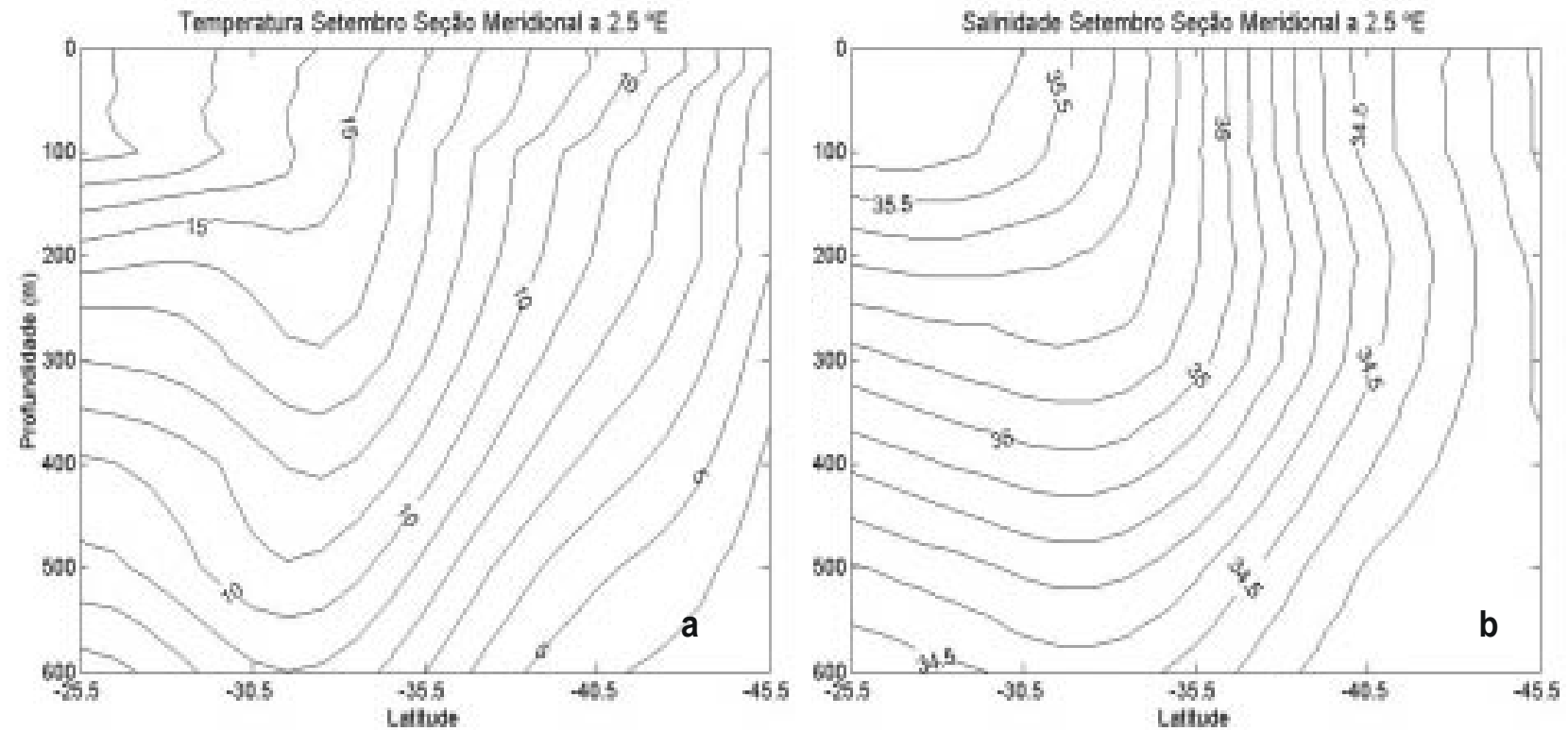

Figura 4 - Seção Meridional a 2,5 $5^{\circ} \mathrm{E}$ (Região Leste) entre $40,5^{\circ} \mathrm{S}$ e $25,5^{\circ} \mathrm{S}$ de (a) temperatura potencial $\left({ }^{\circ} \mathrm{C}\right)$ com intervalo de $1^{\circ} \mathrm{C}$ e $(\mathrm{b})$ Salinidade com intervalo de 0.1 . 

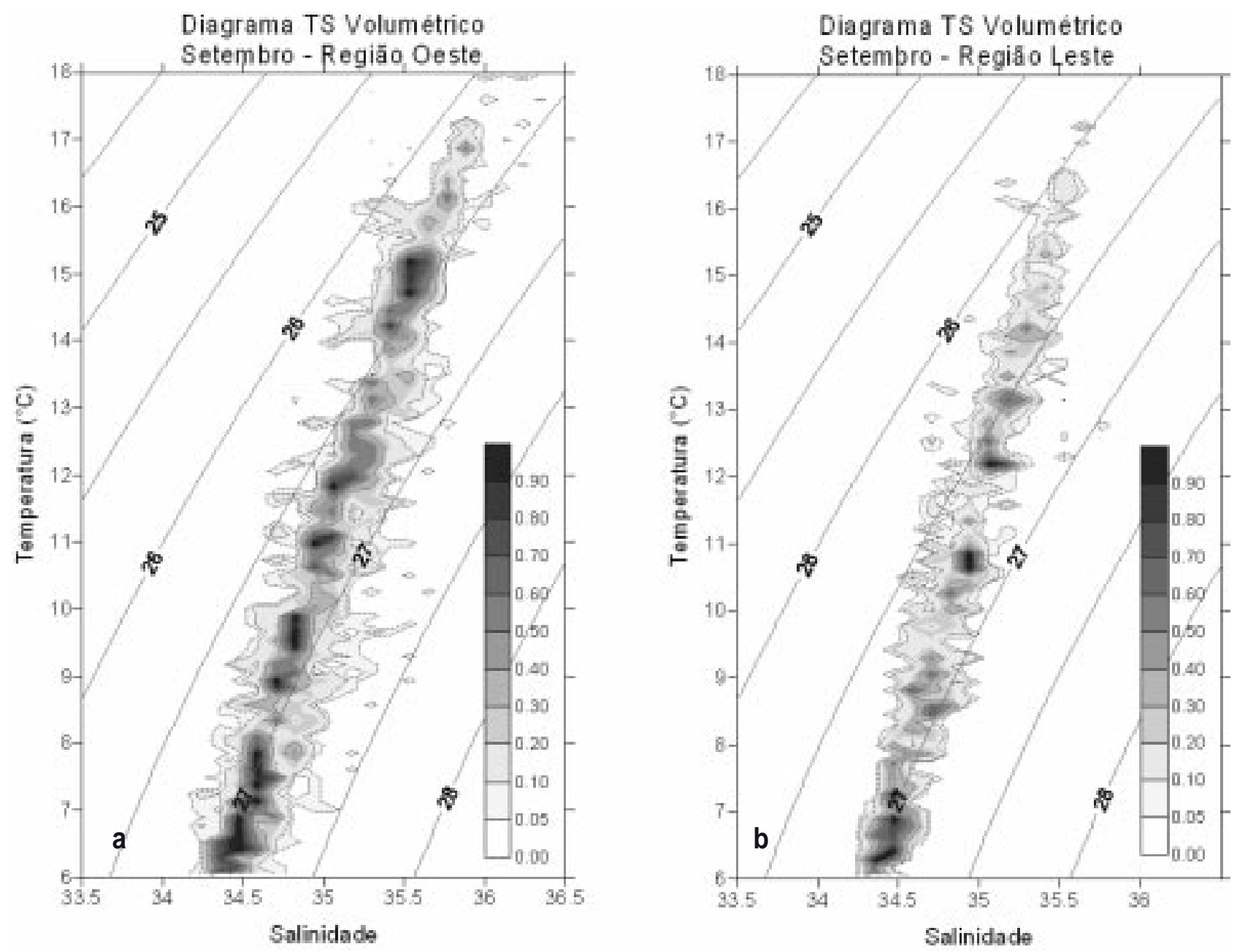

Figura 5 - Diagrama TS volumétrico para as regiões Oeste (a) e Leste (b). Escala de $10^{13} \mathrm{~m}^{3}$.

possivelmente afeta, a representação das águas modais no diagrama TS volumétrico. Apesar das limitações, no presente estudo verificou-se que a base Levitus conservou as feições indicativas de algumas águas modais do Oceano Atlântico Sul, sendo possível identificar termostatas e halostatas presentes na coluna d'água. A utilização desta base para estudos de águas modais pode ser uma alternativa para regiões pouco amostradas ou para épocas do ano restritas ao acesso de cruzeiros oceanográficos.

\section{REFERÊNCIAS}

Hanawa, K. \& Talley, L.D. 2001. Mode waters, p. 373386. In: G. Siedler et al. [Eds.], Ocean circulation and climate: observing and modelling the global ocean. International Geophysics Series.

Hosoda, S.; Xie, S.-P.; Takeuchi, K. \& Nonaka, M. 2001. Eastern North Pacific Subtropical Mode Water in a general circulation model: Formation mechanism and salinity effects. J. Geophys. Res., 106 (C9):19.671-19.681.

Levitus, S. 1982. Climatological Atlas of World Ocean. National Oceanic and Atmospheric Administration, Rockville.

Maamaatuaiahutapu, K.; Provost, C.; Andrié, C. \& Vigan, X. 1999. Origin and ages of mode waters in the Brazil-Malvinas Confluence region during austral winter 1994. J. Geophys. Res., 104 (09):21.05121.061.

Marshall, J.C.; Nurser, A.J.G. \& Williams, R.G. 1993. Inferring the Subtropical Rate and Period over the North Atlantic. J. Phys. Oceanogr., 23:1315-1329.

McCartney, M.S. \& Talley, L.D. 1982. The Subpolar Mode Water of the North Atlantic Ocean. J. Phys. Oceanogr., 12:1169-1188.

Provost, C.; Escoffier, C.; Maamaatuaiahutapu, K.; Kartavtseff, A. \& Garçon, V. 1999. Subtropical mode waters in the south Atlantic Ocean. J. Geophys. Res., 104 (09):21.033-21.049. 
Felix et al:: Águas modais subtropicais.

Roemmich, D. \& Cornuelle, B.1992. The subtropical mode waters of the south Pacific Ocean. J. Phys. Oceanogr., 22:1178-1187.

Silveira da I.C. A.; Schmidt, A.K.; Campos, E.J.D. \& Ikeda, Y. 2000. A Corrente do Brasil ao largo da costa leste brasileira. Braz. Jour. Oceanogr., 48(2):171-183. (CRN - 061)

Williams, R.G.; Spall, M.A. \& Marshall, J.C. 1995. Does Stommel's Mixed Layer "Demon" Work?. J. Phys. Oceanogr., 25:3089-3102. 